\section{Immune cell biology}

\section{INTEGRATION OF HIGH DIMENSIONAL DATASETS IN AN IMMUNOCOMPETENT MAMMARY MOUSE MODEL REVEALS PATHWAYS OF TOLERANCE AND RESISTANCE TO IMMUNE CHECKPOINT BLOCKADE}

${ }^{1}$ Lin Ma, ${ }^{2}$ Jian-Hua Mao, ${ }^{1}$ Mary Helen Barcellos-Hoff, ${ }^{1}$ Jade Moore*. ${ }^{1}$ University of California, San Francisco, San Francisco, CA, USA; '2Lawrence Berkeley National Laboratory, Berkeley, CA, USA

Background Checkpoint inhibitors can induce robust and durable responses in a subset of patients. Extending this benefit to more patients could be facilitated by better understanding of how interacts with immune cells with the tumor microenvironment, which is a critical barrier to control both local and systemic disease. The composition and pattern of the immune infiltrate associates with the likelihood of response to immunotherapy. Inflamed tumors that exhibit a brisk immune cell infiltrate are responsive, while those in which immune cells are completely or partially excluded are not. Transforming growth factor $\beta$ (TGF $\beta$ ) is immunosuppressive and associated with the immune excluded phenotype.

Methods Using an immune competent mammary tumor derived transplant (mTDT) model recently developed in our lab, exhibits inflamed, excluded or deserts immune infiltrate phenotypes based on localization of CD8 lymphocytes. Using whole transcriptome deep sequencing, cytof, and PET-CT imaging, we evaluated the tumor, microenvironment, and immune pathway activation among immune infiltrate phenotypes.

Results Three distinct inflamed tumors phenotypes were identified: 'classically' inflamed characterized by pathway evidence of increased CD8 $+\mathrm{T}$ cells and decreased PD-L1 expression, inflamed tumors with pathways indicative of neovascularization and STAT3 signaling and reduced T cell mobilization, and an inflamed tumor with increased immunosuppressive myeloid phenotypes. Excluded tumors were characterized by TGF $\beta$ gene expression and pro-inflammatory cytokine signaling (e.g. TNF $\alpha$, IL1 $\beta$ ), associated with decreased leukocytes homing and increased immune cell death of cells. We visualized and quantified TGF $\beta$ activity using PET-CT imaging of 89Zr-fresolimumab, a TGF $\beta$ neutralizing antibody. TGF $\beta$ activity was significantly increased in excluded tumors compared to inflamed or desert tumors, which was supported by quantitative pathology (Perkin Elmer) of its canonical signaling target, phosphorylated SMAD2 (pSMAD2). pSMAD2 was positively correlated with PD-L1 expression in the stroma of excluded tumors. In contrast, in inflamed tumors, TGF $\beta$ activity positively correlated with increased $\mathrm{F} 4 / 80$ positive macrophages and negatively correlated with expression of PD-L1. CyTOF analysis of tumor and spleen immune phenotypes revealed increased trafficking of myeloid cells in mice bearing inflamed tumors compared to excluded and deserts.

Conclusions The immunocompetent mTDT provides a model that bridges the gap between the immune landscape and tumor microenvironment. Integration of these high-dimensional data with further studies of response to immunotherapies will help to identify tumor features that favor response to treatment or the means to convert those that are unresponsive.

http://dx.doi.org/10.1136/jitc-2020-SITC2020.0492
493 TIRED AND HUNGRY: A POTENTIAL ROLE FOR CD47 IN T CELL EXHAUSTION

'Levi Mangarin, ${ }^{1}$ Cailian Liu, ${ }^{2}$ Roberta Zappasodi, ${ }^{3}$ Pamela Holland, ${ }^{1}$ Jedd Wolchok, ${ }^{1}$ Taha Merghoub, ${ }^{1}$ Chien-Huan Weng ${ }^{*},{ }^{1}$ Chien-Huan Weng, ${ }^{1}$ David Schroder. ${ }^{1}$ Memorial Sloan-Kettering Cancer Center, New York, NY, USA; ${ }^{2}$ Weill Cornell Medical College, New York, NY, USA; ${ }^{3}$ Surface Oncology, Inc., Cambridge, MA, USA

Background Multiple suppressive mechanisms within the tumor microenvironment are capable of blunting anti-tumor $\mathrm{T}$ cell responses, including the engagement of inhibitory receptors expressed in tumor-associated, exhausted CD8 $+\mathrm{T}$ cells, such as programmed cell death protein 1 (PD-1), T-cell immunoglobulin and mucin-domain containing-3 (TIM-3), lymphocyteactivation gene 3 (LAG-3), 2B4 (also known as CD244), and $\mathrm{T}$ cell immunoreceptor with Ig and ITIM domains (TIGIT). ${ }^{1} 2$ While immune checkpoint blockade therapies aimed at reinvigorating $\mathrm{T}$ cell effector function have demonstrated their clinical effectiveness, ${ }^{3}{ }^{4}$ not all patients demonstrate long-term disease control. ${ }^{5}$ The refractory nature of terminally differentiated, exhausted CD $8+\mathrm{T}$ cells to be reinvigorated by PD-1 blockade is one potential cause. ${ }^{6-8}$ This limitation warrants the need to explore modulatory pathways that potentially program $\mathrm{T}$ cells toward exhaustion.

Methods Single cell-RNA sequencing (scRNA-seq) data derived from the tumor-infiltrating lymphocytes (TILs) of melanoma patients ${ }^{9}$ were used for transcriptomic analysis and flow cytometry results were used to quantify protein levels in TILs. Murine B16-F10 (B16) melanoma model was used for both in vitro and in vivo studies. TCR-transgenic Pmel-1 and OT-1 transgenic mice, as well as CD47-/- (knockout, KO) mice were purchased from the Jackson Laboratory to generate CD47+/+ (wild-type, WT), CD47 \pm (heterozygote, HET) mice with Pmel-1 or OT-1 background. For T cell co-transfer studies, Rag-deficient mice or $\mathrm{C} 57 \mathrm{BL} / 6 \mathrm{j}$ mice with sub-lethal irradiation (600cGy) were used as recipients. Naïve TCR-transgenic CD47-WT and CD47-HET CD8 + T cells were labelled, mixed in a 1:1 ratio for co-transfer experiments.

Results Flow cytometry analysis of human melanoma TILs found a strong upregulation of CD47 expression in tumorassociated, exhausted CD8 + T cells. We confirmed that CD47 transcription is significantly elevated among CD8 $+\mathrm{T}$ cells with a phenotype consistent with exhaustion using scRNA-seq results of TILs derived from melanoma patients. ${ }^{9}$ Our study in murine B16 melanoma model confirms our finding in melanoma patients. To specifically address the role of CD47 in anti-tumor CD8 effector function, we conducted $\mathrm{T}$ cell cotransfer studies and found that CD8 $+\mathrm{T}$ cells with lower copy number of CD47 (CD47-HET) significantly outnumber the cotransferred CD47-WT CD8 + T cells within the tumor, exhibiting an enhanced effector function and less exhausted phenotype. Our study demonstrates a potentially novel role for CD47 in mediating CD8 + T cell exhaustion.

Conclusions CD47 expression in CD8 $+\mathrm{T}$ cells programs $\mathrm{T}$ cells toward exhaustion.

Ethics Approval All mice were maintained in microisolator cages and treated in accordance with the NIH and American Association of Laboratory Animal Care regulations. All mouse procedures and experiments for this study were approved by the MSKCC Institutional Animal Care and Use Committee (IACUC).

\section{REFERENCES}

1. Wherry EJ and M Kurachi. Molecular and cellular insights into T cell exhaustion. Nat Rev Immunol 2015;15(8): p. 486-99. 
2. Thommen DS and Schumacher TN. T Cell Dysfunction in Cancer. Cancer Cell 2018;33(4): p. 547-562.

3. Ribas $A$ and Wolchok JD. Cancer immunotherapy using checkpoint blockade. Science 2018. 359(6382): p. 1350-1355.

4. Sharma $P$ and Allison JP. The future of immune checkpoint therapy. Science 2015; 48(6230): p. 56-61.

5. Sharma $\mathrm{P}$, et al. Primary, adaptive, and acquired resistance to cancer immunotherapy. Cell 2017. 168(4): p. 707-723.

6. Schietinger, A., et al., Tumor-specific $T$ cell dysfunction is a dynamic antigendriven differentiation program initiated early during tumorigenesis. Immunity 2016:45(2): p. 389-401.

7. Pauken KE, et al., Epigenetic stability of exhausted $T$ cells limits durability of reinvigoration by PD-1 blockade. Science 2016;354(6316): p. 1160-1165.

8. Philip $\mathrm{M}$, et al., Chromatin states define tumour-specific $\mathrm{T}$ cell dysfunction and reprogramming. Nature 2017;545(7655): p. 452-456.

9. Sade-Feldman $\mathrm{M}$, et al., Defining T Cell States associated with response to checkpoint immunotherapy in melanoma. Cell 2018;175(4): p. 998-1013 e20.

http://dx.doi.org/10.1136/jitc-2020-SITC2020.0493

\section{BASAL CELL CARCINOMA DEMONSTRATES A T-CELL EXCLUSION IMMUNE PHENOTYPE IN CONTRAST TO OTHER ANTI-PD-1 THERAPY RESPONSIVE CUTANEOUS MALIGNANCIES}

${ }^{1}$ Geoffrey Gibney ${ }^{*},{ }^{2}$ Joanne Xiu, ${ }^{3}$ Gino In, ${ }^{4}$ Steven O'Day, ${ }^{5}$ Jose Lutzky, ${ }^{6}$ Joseph Drabick, ${ }^{7}$ Ari VanderWalde, ${ }^{2}$ Kelsey Poorman, ${ }^{2}$ W Korn, ${ }^{1}$ Michael Atkins. ${ }^{1}$ Georgetown University LCCC, Washington, DC, USA; ${ }^{2}$ Caris Life Sciences, Phoenix, AZ, USA; ${ }^{3}$ Norris Comprehensive Cancer Center, Los Angeles, CA, USA; ${ }^{4}$ John Wayne Cancer Institute, Santa Monica, CA, USA; ${ }^{5}$ UM Sylvester Comprehensive Cancer Center, Miami, FL, USA; ${ }^{6}$ Penn State Hersey Cancer Institute, Palmyra, PA, USA; ${ }^{7}$ West Cancer Center, Germantown, TN, USA

Background Basal cell carcinoma (BCC) is considered an immunogenic tumor based on the high tumor mutational burden (TMB), increased incidence in immunocompromised patients, and responsiveness to imiquimod, a toll-like receptor agonist therapy. However, anti-PD-1 immunotherapy response rates in patients with advanced BCC appear less than that seen with other advanced cutaneous malignancies. Molecular profiles of BCC tumors were analyzed to determine immune phenotypes and resistance mechanisms in comparison to other anti-PD-1 therapy responsive cutaneous malignancies.

Methods Next generation sequencing on DNA (NGS; NextSeq and Novaseq), PD-L1 immunohistochemistry (SP-142 and 288 antibody clones, cutoff $>5 \%$ tumor staining) and mRNA gene expression level (Whole Transcriptome Sequencing, NovaSeq) data from BCC $(\mathrm{N}=69)$, melanoma $(\mathrm{N}=914)$, and cutaneous squamous cell carcinoma (SCC) tumors $(\mathrm{N}=165)$ at Caris Life Sciences (Phoenix, AZ) were analyzed. Tumor mutational burden (TMB) was calculated by counting all non-synonymous missense mutations that had not been previously described as germline alterations. Microenvironment cell population counter ${ }^{1}$ was used to estimate cell population abundance in the TME. Gene set enrichment analysis (GSEA) was performed on transcriptomes. ${ }^{2}$ Statistical significance was set at $\mathrm{P}$ value or false discovery rate $(\mathrm{FDR})<0.05$.

Results Of the 69 BCC tumors with NGS data, the most frequent mutations were in PTCH1 (82\%), P53 (73\%) and ARID1A (42\%); additional relevant mutations included SMO (18\%), JAK1 (9\%), PI3KCA (6\%), APC (4\%), and CTNNB1 $(3 \%)$. TMB was significantly greater in BCC compared to melanoma (median 30.5 vs $12 \mathrm{mut} / \mathrm{Mb}, \mathrm{P}<0.0001$ ) and similar to SCC (median $29.5 \mathrm{mut} / \mathrm{Mb}, \mathrm{P}=0.9389$ ). PD-L1 positivity was $1 / 23(4 \%)$ in BCC, $215 / 831(26 \%)$ in melanoma, and $81 / 147(56 \%)$ in SCC. Interferon gamma and T-effector immune gene analyses showed significantly lower expression in BCC compared to melanoma and SCC (e.g., IFNg
$\mathrm{TPM}=0.26$ (BCC) vs 0.65 and 0.58 (melanoma, SCC, both $\mathrm{P}<0.01$ ). BCC demonstrated the lowest CD-8 T-cell fractions and the highest neutrophil and cancer associated fibroblast (CAF) fractions compared to melanoma and SCC. Angiogenesis and TGF-beta gene sets were enriched in BCC compared to melanoma $(\mathrm{NES}=1.5, \quad \mathrm{FDR}=0.046$ and $\mathrm{NES}=1.35$, $\mathrm{FDR}=0.055$, respectively), but not compared to SCC $(\mathrm{NES}=0.90, \quad \mathrm{FDR}=0.57 \quad$ and $\quad \mathrm{NES}=0.94, \quad \mathrm{FDR}=0.60$, respectively).

Conclusions While BCC tumors demonstrated a high TMB, a markedly lower level of adaptive anti-tumor immunity in comparison to other cutaneous malignancies was observed. T-cell exclusion mechanisms mediated through CAFs and desmoplasia, with upregulation of TGF-beta and angiogenic signaling, may play a role. Further investigation into abrogation of these mechanisms is warranted to develop improved anti-PD-1 based therapies for BCC.

\section{REFERENCES}

1. Becht E, Giraldo NA, Lacroix L, Buttard B, Elarouci N, Petitprez F, Selves J, Laurent-Puig $P$, Sautès-Fridman C, Fridman WH, de Reyniès A. Estimating the population abundance of tissue-infiltrating immune and stromal cell populations using gene expression. Genome Biol 2016; 17(1):218.

2. Subramanian A, Tamayo P, Mootha VK, Mukherjee S, Ebert BL, Gillette MA, Paulovich A, Pomeroy SL, Golub TR, Lander ES, Mesirov JP. Gene set enrichment analysis: a knowledge-based approach for interpreting genome-wide expression profiles. Proc Natl Acad Sci U S A 2005;102(43):15545-50.

http://dx.doi.org/10.1136/jitc-2020-SITC2020.0495

\section{IMMUNE CELL PROFILING ACROSS SOLID TUMOR TYPES BY MASS CYTOMETRY REVEALS TUMOR ENRICHMENT OF PD-1+/LAG-3+ CD8 MEMORY T CELLS THAT EXHIBIT TUMOR-REACTIVE YET DYSFUNCTIONAL FEATURES}

Bradley Garman, Laurence Menard, Can Jiang, Sherif Daouti, Priyanka Mehta, Miye Jacques, Mohan Bolisetty, Christos Hatzis, Nataly Manjarrez Orduno, Michaela Bowden, Justin David*, Justin David, Justin David. Bristol Myers Squibb, Lawrenceville, NJ, USA

Background Characterization of human immune responses by profiling immune cells from patients is critical for the successful development of immuno-oncology agents and is useful to understand mechanism-of-action, identify pharmacodynamic/ response biomarkers, and guide patient selection strategies. Extensive immune cell heterogeneity necessitates comprehensive high parameter immunophenotyping to yield these actionable insights.

Methods Cytometry by time-of-flight (CyTOF) was performed on homogenates from commercially procured tumors $(n=28)$ and matched PBMCs $(n=7)$ from patients with various solid tumors (colon $(n=10)$, endometrial $(n=9)$, kidney $(n=4)$, liver $(n=2)$, skin $(n=1)$, lung $(n=1)$, and gastro-intestinal $(n=1))$. Two antibody panels, recognizing a total of 18 lineage and 31 target proteins, were used to profile marker expression among the major lymphocyte and myeloid lineages. Data were analyzed using manual gating and non-linear dimensionality reduction (tSNE and UMAP), and expression was measured by frequency ( $\%$ gate) and arcsinh-transformed median ion counts. Pairwise Wilcoxon Rank Sum tests were performed on arcsinh-transformed median ion counts to determine statistically significant differences in marker expression, and $\mathrm{P}$ values were adjusted using Benjamini-Hochberg correction $(\mathrm{p}<0.05$ considered statistically significant). Cell subpopulation percentages were compared using unpaired two-sided T-tests. Sample populations with less than 150 events were excluded from 